\title{
Vertical Axis Wind Turbine: A Novel Approach to Development and Modeling
}

\author{
Md. Hafizul Imran \\ Department of Software \\ Engineering \\ Daffodil International University
}

\author{
Rony Shaha \\ Department of Software \\ Engineering \\ Daffodil International University
}

\author{
Rifat Bin Mahi \\ Department of Information and \\ Communication Engineering \\ University of Electronics Science \\ \&Technology of china
}

\author{
Md Rokon Ujjaman \\ Department of Electrical and Electronics Engineering \\ Daffodil International University
}

\begin{abstract}
When the wind blows towards the vertical axis wind turbine, it strikes the blades of the wind turbine, causing the blades of the wind turbine to move. The downside of this turbine where magnetic flux creating in between the upper and bottom of the magnet which the magnets and transformers act as a generator due to the north and south pole of the transformer and trans to generating electrical energy. Instead of using the two big neodymium magnets in the lower part of the wind turbine, rather set up a bearing to rotate the turbine blades which leads to rotating the turbine with a minimum level airflow. This paper affords the well-shaped design and implementation of this turbine and developed blades profiled ring model around it, subsequently generalize the output power, will assist the residential area to produce renewable energy where the airflow is not high enough.
\end{abstract}

\section{Keywords}

Vertical axis, wind turbine, magnetic flux, neodymium magnet

\section{INTRODUCTION}

Electricity generation possession by oil, coal, and other fossilfuel and its perverts the environmental natural conditions, which contemplate world scientists and leaders to overcome this phenomenon. Human actions, especially the greenhouse emissions effect, are causing the earth to become one-degree centigrade hotter in landmasses, which is due to the human daily reactions to the earth [1]. To reduce these emissions of the greenhouse effect, the allegiance of the fossil-fuel should dismissal to the renewable resources which can take technology to the final stage of the energy supply Last two decades, the scientist is reliance to the renewable energy, where wind energy is one of them to integrate energy generation one step forward. The wind power revolution began about 5000 years ago, while many cultures in recorded existence often use wind power to generate kinetic energy or for the navigator.

Wind Turbine, where wind power relocates this energy to electrical form [3] using it to fulfill electricity demand and minimize fossil-fuel consumption. Wind turbine elevation has improved 10 times as much in the last 2 centuries, while it was reduced energy cost of its life-cycle [2]. In 1700 B.C Babylonian Dynasty was used windmills to supply water to the land for agriculture. Later this was proven in the seventh century when it utilizes in vertical axis with drag element of wind energy to water pumping and squeezing in Iran and Afghanistan [4]. Wind turbines fundamentally two types, can spin into two-axis, horizontal and vertical, while horizontal one called Horizontal Axis Wind Turbine (HAWT) and vertical one called Vertical Axis Wind Turbine (VAWT). The structure of horizontal is wing shape and vertical is egg beater shape. Though, HAWT is extensively employed these days because of the orthogonal foundation. Nevertheless, VAWT has a massive achievement to transform electricity from wind power in suburban places.

The tremendous development of VAWT has been done by 1922-31 by engineers Savonius [7] and Darriues[5], introduces two types of such as banded and straight blade[6]. They invented different shape of the rotor which is Darriues rotor, Darriues H-rotor, and Savonius rotor.

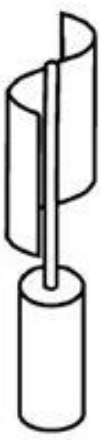

(A)

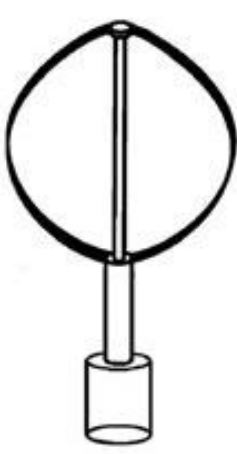

(B)

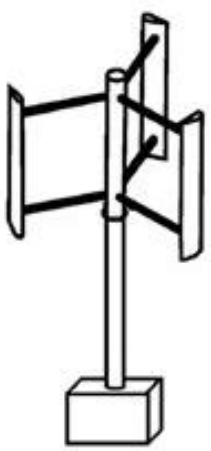

(C)
Figure 1. The rotor of VAWT. (A) Savonius (B) Darriues (C) H-shape [8]

This paper will think about the unwavering quality, or all the more the instability, and will present the development of vertical axis wind turbines and a novel approach to their developments and implementations.

\section{METHODOLOGY AND MODELING}

This segment presents and gives a short depiction of the significant parts and factors that will add to a proficiently working breeze turbine. These variables are airpower, the generator. The later segments would give a top to bottom investigation of the embodiment of each factor and its 
capacity and significance to the general activity of a vertical pivot air-powered turbine.

\subsection{Power of Wind}

Doubtlessly, the funeral director's efficiency to work only relies on the energy of the air as well as its accessibility. The air is familiar with other types of sun-situated imperativeness since it works out as intended on account of the unbalanced warming of our climate by the sun joined with hypothetical location details of the world's face. Two types of the breeze are important to the operations of wind turbines: regional and galactic winds. That is indeed the more essential, although it is frequently a key element in the location of highly efficient wind turbines, specifically those that have a horizontal axis.

These sorts of breezes are normally constructed towards shorelines, hilltops, grassy fields, and vale. Previously, the sort you'll see in daily surroundings including towns or rural regions, essentially everywhere there are people. This form of wind isn't ideal for producing electricity; it would only be useful whenever it's followed by terrestrial air that is traveling. Whenever a certain amount of wind will flow through the turbine blades then the rotation will occur continuously, where energy was calculated by the area and respect of the velocity as named kinetic energy which expresses by $\mathrm{K}$,

$$
\mathrm{K}=1 / 2 \times \text { Mass } \times(\text { Velocity }) 2
$$

Mass is defined by kilogram and velocity by a meter per second. The density of the wind power nearly $1.23 \mathrm{~kg} / \mathrm{m} 3$. When the wind impact the blade, every second has provided an equation,

$$
\text { Mass }=\text { Density } \times \text { Velocity } \times \text { Area }
$$

Nevertheless, the amount of the wind when hit the area of the blade in every second then the final equation is,

$$
\text { Kinetic Energy }=1 / 2 \times \text { Density } \times \text { Area } \times(\text { Velocity }) 3
$$

The strength of the wind and efficient ways to build wind turbines for optimum wind energy will be addressed in greater detail in the subsequent chapter. A complete portfolio is shown below figure [10].

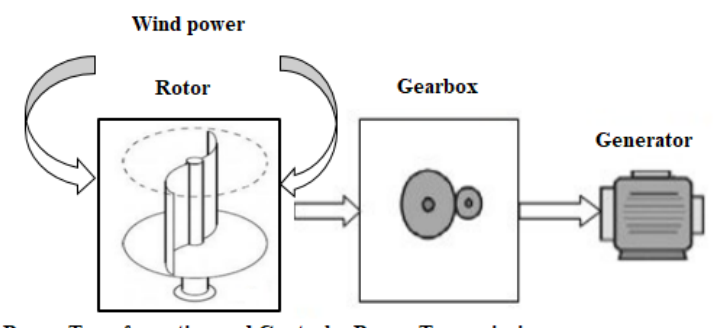

Power Transformation and Control Power Transmission

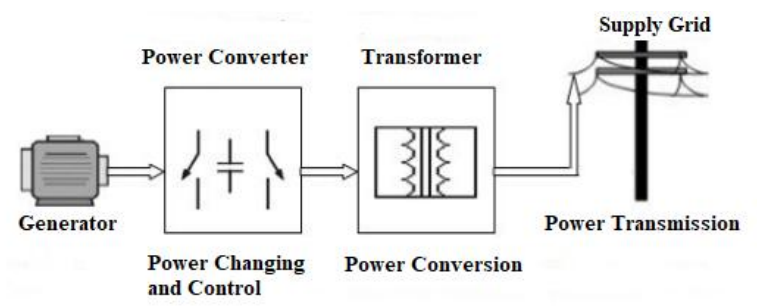

Figure 2. Wind turbine electro-mechanical Process

\subsection{Generator}

A generator, in its most fundamental form, transforms mechanical power into electrical power. It is widely used in a variety of systems, so these frameworks, almost have certain connections. Nevertheless, a couple of distinctions exist which genuinely perceives structure dealing with a motor. With the center point movement generator plan, its operability relies upon unending alternators of the magnet, in which the principle of magnetic field and the magnets are the most critical variables in generator operation. The air gap area is identical at the spinning axis, where this gap produces the perpendicular direction of the magnetic fluxes toward these generators. In additional parts, it will explore their fundamental movement and the arrangement of our plan. Helpful for convincing power age; it conceivably possesses a high value whenever it goes with the breeze planetary movement. In future areas, much spotlight will determine the force of the breeze and reasonable medium to deal with setup wind turbines owing to ideal breeze energy creation[11].

\subsection{Generation of Power}

A generator is a basic thing to have a solid handle of the key laws that control its introduction. A nearby shifting magnetic field is needed to cause a voltage in a cable. The induced voltage proportional to the field density of magnitude and on the region of the spindle. Flux $(\Phi)$ is a term that describes the interaction within field density and the area. The generator configuration defines how this flux changes over time. A voltage is produced by the axial flux generator by adjusting the magnetic flux. Voltage conveyed by every twist will be resolved conducting Faraday's law of acknowledgment

$$
V=-N(d \varnothing / d t)
$$

\subsection{Induced Electro-Magnetic Force}

The components producing an electromotive force or voltage must be explained to understand how an axial stream generator is constructed. The magnetic field produces an induced EMF, to produce a magnetic field voltage, Michael Faraday conducted experiments with stable currents and a basic transformer. He found that a continuous magnetic field cannot cause a voltage, but may cause a time difference. This was a crucial development of so-called electromagnetic induction, a discovery that is essential to a generator design. The relative movement of a magnetic field that produces a tension enables one to be innovative in electricity production.

\subsection{Magnetic Flux}

The degree of the magnetic flux is the most vital when the twist in an alluring field inverse to the field. The arrangement of a center progress generator, the ideal to keep the twists inverse to the field conveyed by the enduring magnets. Various standards of motors, a bending turn inside an alluring field. The winding number is raised because every winding is placed at the edge of $90^{\circ} \mathrm{C}$ throughout the field.The direction of the coils to the surface does not shift in our nature, rather it is time-varying. The law of induction in Faraday states that the electrical field induced is proportional to the change in the flow of magnetism throughout time.

$$
V=-d \varnothing / d t
$$

\subsection{Revolving Field}

The arrangement of turning field generators is to have the armature part on the stator and the alluring field part on the rotor. A key arrangement of turning field single-stage generators shows up on the right. There are two appealing poles, north and south, annexed to a rotor and two twists 
which are related in course of action and correspondingly isolated on the stator. The windings of the two circles are in reverse making a beeline for having the current to stream likewise because the two twists reliably speak with negating polarities. Since posts and twists are also isolated and the territories of the shafts match the regions of the circles, the appealing lines of intensity are cut at a comparative whole at any degree of the rotor. Accordingly, the voltages started to all windings have a comparative impetus at some irregular time. The voltages from the two twists are "in stage" to each other. As such, the total yield voltage is on numerous occasions the voltage affected in each winding. In the figure, at the position where shaft number 1 and twist number 1 meet, the generator conveys the most raised yield voltage on one bearing. As the rotor turns 180 degrees, the yield voltage is subbed to make the most important voltage on the other heading. The repeat of the forced air system yield for the present circumstance counterparts to the number of turns of the rotor consistently.

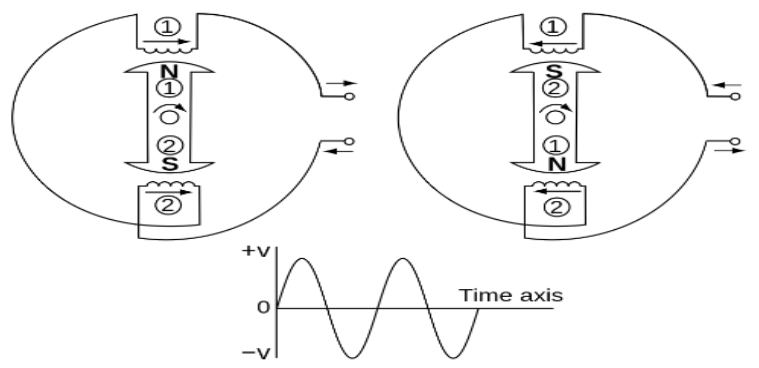

Figure 3. Revolving Field Ac Input

This plan can likewise permit us to expand the yield recurrence by including more posts. In this model on the right, having 4 curls associated in arrangement on the stator and the field rotor has 4 shafts. The two loops and posts are similarly dispersed. Each shaft has an inverse extremity to its neighbors which are calculated at 90 degrees. Each curl likewise has inverse twisting to its neighbors. This design permits the lines of power at 4 posts to be cut by 4 curls at a similar sum at a given time. At every 90-degree pivot, the voltage yield extremity is changed from one course to the next. Along these lines, there are 4 patterns of the air conditioner yield in one pivot. As the 4 curls are wired in arrangement and their yields are "in stage", the air conditioner yield of this single-stage generator will have multiple times the voltage produced by every individual loop.

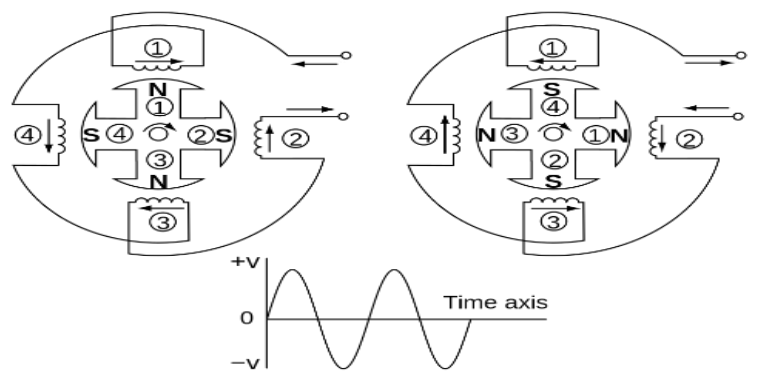

Figure 4. Revolving Field

An advantage of the spinning field configuration is that on the off chance that the shafts are lasting magnets, at that point there is no compelling reason to utilize any slip ring and brush to convey power out of the generator as the curls are fixed and can be wired legitimately from the generator to the outer burdens.

\subsection{Magnetic Selection}

It is important to consider the properties of the magnets and the various sizes, styles, and substances. There have been four groups of commercially produced magnets, each of which has its own magnetically characteristics depending on its molecular structure.

Alnico, ceramic, samarium cobalt, and neodymium iron boron are all classified as Nd-Fe-B in four distinct groups. Nd-Fe-B is the latest addition to this catalog of industrial materials and also the best characteristics with all conductive particles are exhibited at ambient temperature. In the B-H diagram demonstrate in figure $4, \mathrm{Nd}-\mathrm{Fe}-\mathrm{B}$ has a quite prominent magnetic feature that provides the capacity to withstand deformation and high flux density. This characteristic is very significant since the load is heavy and moving at incredible velocities and has a strong centrifugal acceleration in this direction.

The structure and dimension of its magnet, which would also be closely tied to the positioning of the magnets, are the next element to be taken into consideration. Levitation appears to be the most powerful on the medium axis side, on which the wind turbine total mass is located under an equally spaced load depict in figure 4. This outcome indicates a fundamental depiction of the incorporation of the maglev into its layout. Unless the magnets are constructed like a circle they can conveniently slide down the shaft and face one another with a similar end.

It will allow the repellents to accommodate the wind turbine's strength and size and decrease the number of magnets required to finish the idea.

For this use, the magnets N42 were the permanent magnets selected. These constant Nd-Fe-B circle magnets are studded with nickel to stabilize and shield the magnet inside. The magnets have acceptable in size, external $40 \mathrm{~mm}$ radius, 20 $\mathrm{mm}$ inner radius, and $10 \mathrm{~mm}$ of length.

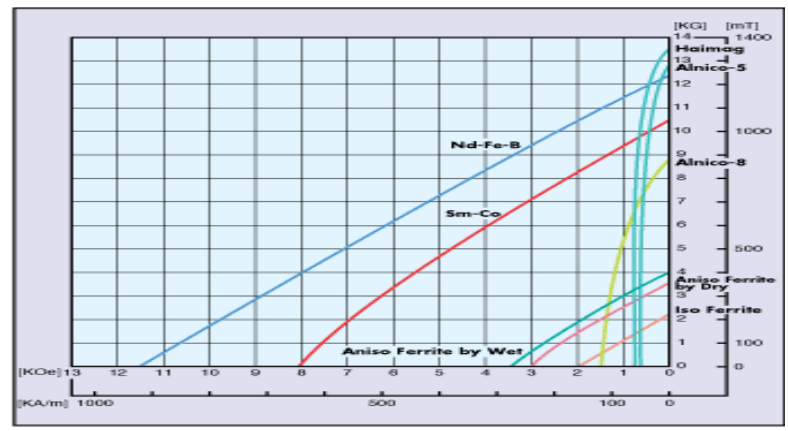

Figure 5. B-H Curve of Magnets ${ }^{[9]}$

\subsection{D Parts layouts of Wind turbine}

In this section, a 3D design of the wind turbine has been introduced, where is divided into four major parts Wind Turbine rotating frame, magnet, Disk, and the shaft of VAWT.
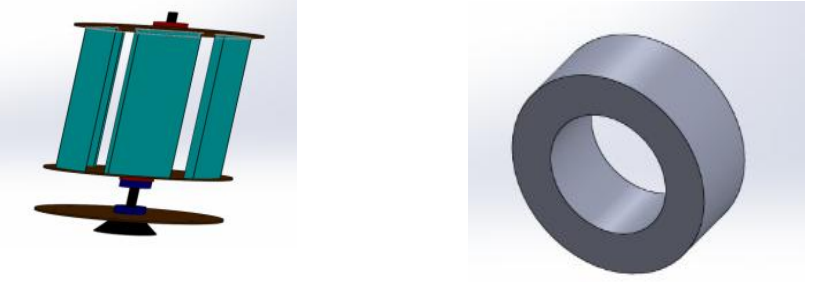


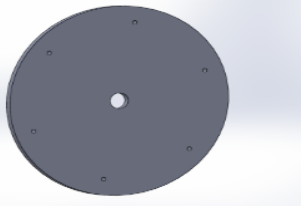

(c) Disk

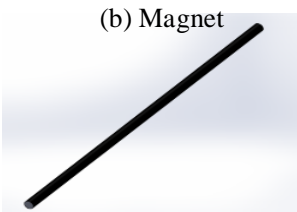

(d) Shaft
Figure 6. The major segment of VAWT

\section{IMPLEMENTATION}

This section is devoted to reflecting how examinations and usage had been done and used in planning the model of the keen success turbine with Neodymium magnets. After serious exploration of all the accessible procedures and segments, to meet the necessity of the ideal force yield and to fulfill the prime goal of the undertaking, reasonable strategies and segments were chosen and actualized both equipment and electrical parts.

\subsection{Mechanical Implementation}

To actualize a shrewd and solid breeze turbine there ought to consummate mix of mechanical and electrical parts. Our breeze turbine has some necessary mechanical parts. In this segment insights concerning planning and usage of the mechanical part will be exhibited bit by bit.

\subsection{Blade Design and implementation}

In this wind turbine, three sharp edges has been utilize, since six edges have the most extreme region inclusion and wind stream which will make this model accomplish enough force coefficient to create power. From the outset, sharp edge was configuration utilizing strong works. As per the plan, it made our cutting edge utilizing UPVC pipe.

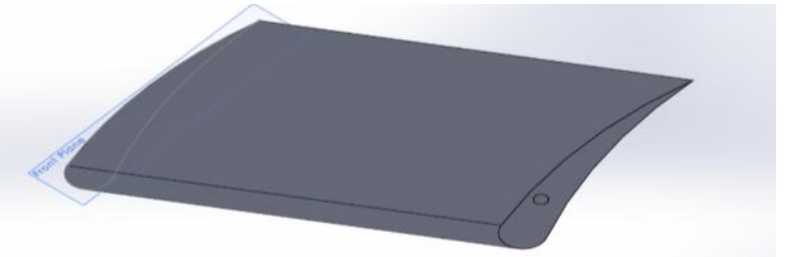

Figure. 7 (a) Side view of the designed blade.

The benefit of using PVC pipe is due to its radial shape itgetting almost aerodynamic shape automatically. Blade length is 18 inch and width is $11 \mathrm{~cm}$. The average weight is around $151 \mathrm{gm}$.

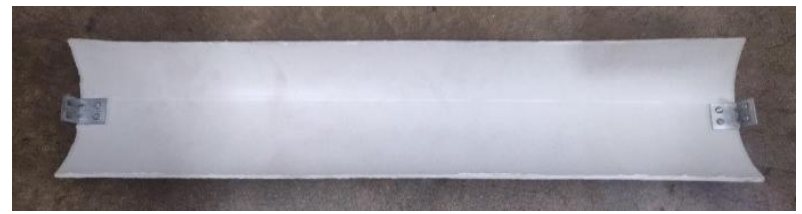

Figure. 7 (b): Real View of the blade.

Then those six blades are added to a two-disc shape plate at $60^{\circ}$ separated. After that, the discs with the blade are inserted into the shaft.

\subsection{Magnet placement}

There are 12 Neodymium magnets are joined with the base circle lower partition. Likewise, disk magnets with $10 \mathrm{~mm}$ width and $4 \mathrm{~mm}$ height are set along its $40 \mathrm{~cm}$ rotor periphery as a North and South Pole one by one. These magnets have the beneficial flow to generate electricity. They are put at a 30-degree point distance in the middle of them.

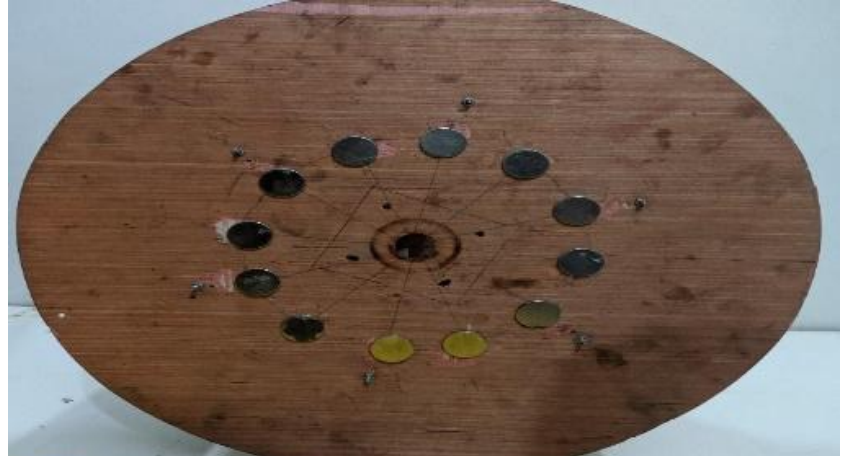

Figure. 8. Magnet placement

\subsection{Coil design and placement}

The fixed turn's number, which is hard to build for each coil. Many turns maximize the emf produced by every coil, however, they also extend the length of each coil. Wire with a larger gauge may be useful to reduce the scale. When the wire radius is considerable, a tiny volume of current flows, causing the wire to warm up due to higher resistance that is another challenging process. While arranging a generator for a given application, the conviction should be understood that the issue got together with tremendous circle shape is responsible for the density of the field. The extension in twist length fabricates opening within two magnets provoking a decline in alluring movement. From now on key voltage and current should be changed. The Twist position is figured out on the stator plot unequivocally inside the plate magnets set on the rotor. Each circle is kept in a course of action to get the most noteworthy yield voltage. The construction was developed by the wire used to turns of one twist is of 42 measure have 2000 turns. 6 courses of action of twists are engineered in the game plan relationship in the model. The twist approach is showed up in figure [9].

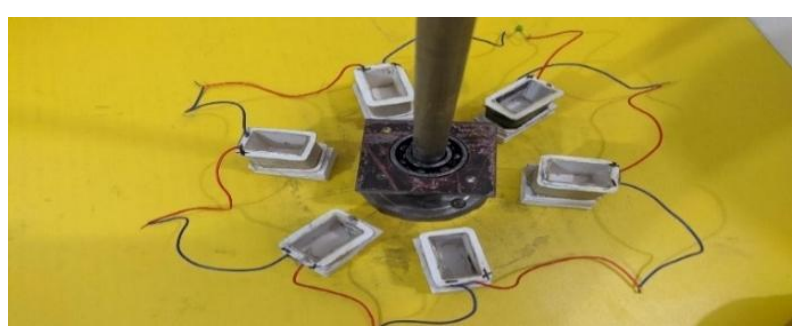

Fig. 9 Placement of coil.

\subsection{The gap between Coil and magnets}

The gap between rotor fixed magnets and the stators coils is 1 $\mathrm{cm}$. At the top of the frame, a bearing has been used to rotate the turbine, instead of using two large neodymium to hold the rotating frame.

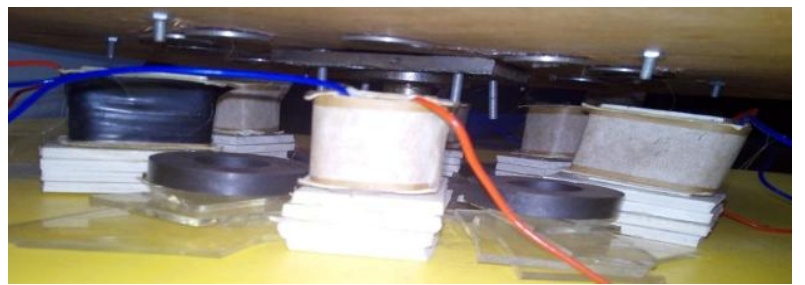

Fig. 10 Gap between rotor and stator

\subsection{Overview of the implemented design}

After, insert all the components than the outcome depicted below the figure. 


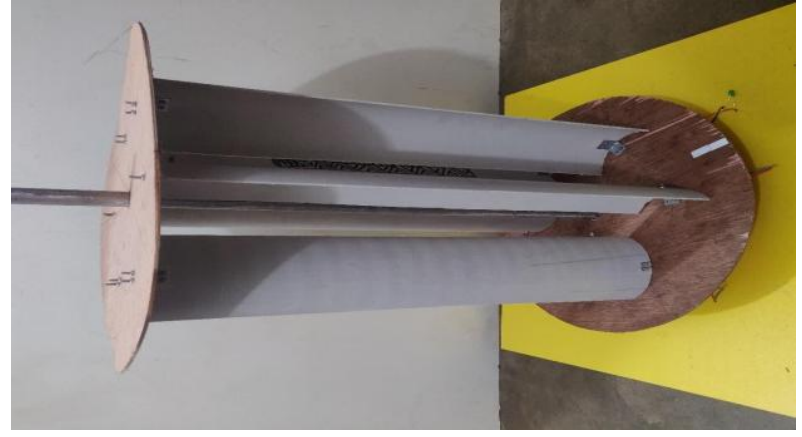

Fig. 11. Vertical axis wind turbine

\section{RESULTS ANALYSIS AND CRITICAL DESIGN REVIEW}

\subsection{Result Analysis}

After planning the parts and structures wanted for testing power yield for the air turbine plans and the structures that wanted to be tried, made the test set-ups needed to test the models and structures. The viability of the items that were fabricated, then it tests to assess. The test setup was in an open region. As our model of a breeze, the turbine isn't working appropriately in typical breeze speed then need to gather the current and voltage esteem for the comparing generator RPM. At that point, the wind speed has been computed for those comparing RPM. How the vibrations from the turbine influence the anxiety on a tree structure are additionally tried to the force yield of the turbine sharp edges and assessed. The strategy of ascertaining the force is tallying the voltage.

Now,

$$
P=V \times I
$$

Where, $P=$ Power in watt

$$
\begin{gathered}
V=\text { Voltage in volt } \\
I=\text { Current in ampere }
\end{gathered}
$$

From the graph, result is clearly shown that voltage and current are respectively dependent on turbine RPM, which is demonstrate Figure 12.

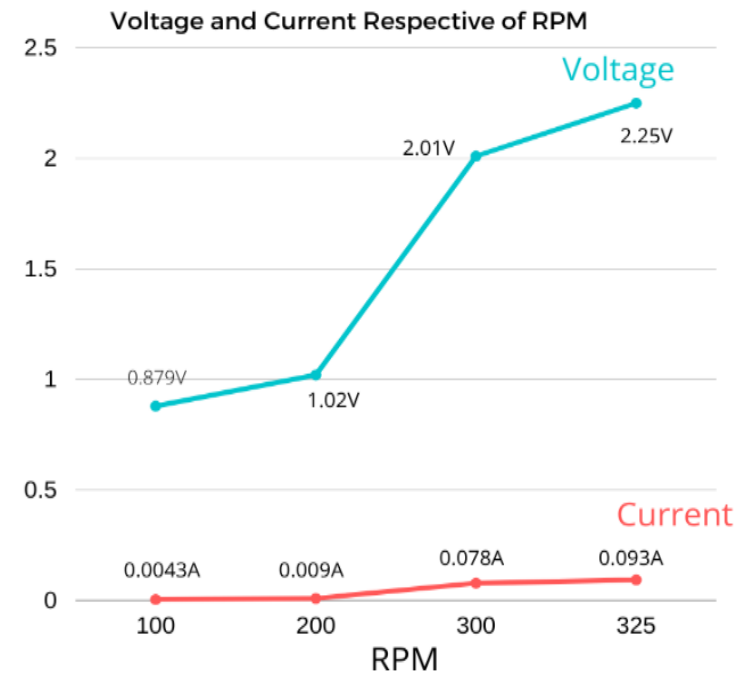

Fig.12. Voltage and Current graph respective to RPM
When the Rotor moves fast (as shown 325rpm) it gives the highest power output (Voltage x Current). In this case it approx. $0.209 \mathrm{~W}$. And the output result is very low when

Table.1. Experimental data of implemented design

\begin{tabular}{|c|c|c|}
\hline RPM & $\begin{array}{c}\text { Wind } \\
\text { Speed } \\
\boldsymbol{m} / \boldsymbol{S}\end{array}$ & $\begin{array}{c}\text { Power } \\
\text { (Watt) }\end{array}$ \\
\hline 100 & 2.2 & 0.0037 \\
\hline 200 & 2.29 & 0.0091 \\
\hline 300 & 2.37 & 0.156 \\
\hline 325 & 2.45 & 0.209 \\
\hline
\end{tabular}

The rotor spins very slowly. Here is a comparison data table no 1 that shows all the output data.

\section{CONCLUSION}

The performance of the turbine is increased through the use of magnets, which enable the turbine to rotate at a great velocity with little turbulence by canceling out the tension mostly on the shaft. In comparison to traditional wind turbines, this proposed format produces more energy with better productivity. The shaft was provided to prevent the rotor from vibrating.

A typical windmill serves 1000 windmills energy which can cover 500000, where a set of maglev turbine covers 750000 houses. 1000 windmills take lots of space (approximately 65000 acres), while maglev windmill takes fewer than hundred acres, which reminds that the comparison between this two types of the turbine maglev wind turbine is quite beneficial rather than others.

\section{REFERENCES}

[1] Coriolis.Energy,"http://coriolis energy.com/wind_energy/wind_index.html, 2013

[2] Amano RS. Review of wind turbine research in 21st century. Journal of Energy Resources Technology. 2017 Sep 1;139(5).

[3] Li Y. Straight-bladed vertical axis wind turbines: history, performance, and applications. InRotating Machinery 2019 Mar 1. IntechOpen.

[4] Golding, Edward William. "Generation of electricity by wind power." (1976).

[5] Marie DG, inventor; Leblance and Vickers Maurice sa, assignee. Turbine having its rotating shaft transverse to the flow of the current. United States patent US 1,835,018. 1931 Dec 8.

[6] Paraschivoiu I. Wind turbine design: with emphasis on Darrieus concept. Presses inter Polytechnique; 2002.

[7] Wenehenubun F, Saputra A, Sutanto H. An experimental study on the performance of Savonius wind turbines related with the number of blades. Energy procedia. 2015 Apr 1;68:297-304.

[8] Khammas F, Mustaffa MT, Ryspek U, Askar K, Qasimr A, Quadir G. Investigate the effect of Differeny Design Parameter on Performance Evaluation of Straight Blade vertical Axis Wind Turbine.International Journal of 
Engineering Technologies and Management Research. 2015 Oct 31;2(4):9-18.

[9] Vaidya H, Chandodkar P, Khobragade B, Kharat RK. Power Generation Using Maglev Windmill. International Journal of Research in Engineering and Technology. 2016 Jun 25;5(06):2319-1163.
[10] Sarkar A, Behera DK. Wind turbine blade efficiency and power calculation with electrical analogy. International Journal of Scientific and Research Publications. 2012 Feb;2(2):1-5.

[11] John M, John R, Syamily PS, Vyshak PA. Maglev windmill. International Journal of Research in Engineering and Technology. 2014 May; 3(05). 